\title{
Adjusting the melting point of a model system via Gibbs-Duhem integration: Application to a model of aluminum
}

\author{
Jess B. Sturgeon and Brian B. Laird* \\ Department of Chemistry and Kansas Institute for Theoretical and Computational Science, University of Kansas, \\ Lawrence, Kansas 66045 \\ (Received 15 June 2000)
}

\begin{abstract}
Model interaction potentials for real materials are generally optimized with respect to only those experimental properties that are easily evaluated as mechanical averages [e.g., elastic constants (at $T=0 \mathrm{~K}$ ), static lattice energies, and liquid structure]. For such potentials, agreement with experiment for the nonmechanical properties, such as the melting point, is not guaranteed and such values can deviate significantly from experiment. We present a method for reparametrizing any model interaction potential of a real material to adjust its melting temperature to a value that is closer to its experimental melting temperature. This is done without significantly affecting the mechanical properties for which the potential was modeled. This method is an application of Gibbs-Duhem integration [D. Kofke, Mol. Phys. 78, 1331 (1993)]. As a test we apply the method to an embedded atom model of aluminum [J. Mei and J.W. Davenport, Phys. Rev. B 46, 21 (1992)] for which the melting temperature for the thermodynamic limit is $826.4 \pm 1.3 \mathrm{~K}$-somewhat below the experimental value of $933 \mathrm{~K}$. After reparametrization, the melting temperature of the modified potential is found to be $931.5 \pm 1.5 \mathrm{~K}$.
\end{abstract}

\section{INTRODUCTION}

The ability of a simulation to successfully predict the properties of real materials is primarily dependent upon the accuracy of the model interaction potential used. The construction of model interactions generally involves the optimization of the parameters of the potential with respect to mechanical properties of the material (crystal lattice constants, elastic constants, liquid density, etc.) as determined from experiment or $a b$ initio calculations. Nonmechanical properties (i.e., those not obtainable as local averages over coordinates) such as phase transition temperatures are difficult to include in such optimization procedures and are generally calculated for the optimized model a posteriori, and the agreement of such quantities with experiment is not guaranteed. However, for some applications that deal directly with such properties, such as in the study of solid-liquid interfaces ${ }^{1}$ in which the melting temperature plays an obviously important role, it is desirable to develop efficient procedures for including such nonmechanical properties in the optimization. Recently, Errington and Panagiotopoulos ${ }^{2}$ have developed a method in which histogram reweighting grand canonical Monte Carlo techniques are used to optimize the parameters of model potentials with respect to vapor-liquid coexistence data, but such procedures are not well suited for optimizations involving solid-liquid coexistence properties, such as the melting temperature. In this work, we outline a general procedure for adjusting the potential parameters for a system designed to model a real system to improve the agreement of the melting point of that system with the experimental value. As an example we present an application to an existing embedded atom model of aluminum. ${ }^{3}$

Our reparametrization scheme is based on the powerful Gibbs-Duhem integration method developed by Kofke. ${ }^{4,5}$ In this technique, the derivative along the coexistence curve of any coexistence condition (such as melting temperature or pressure) with respect to any parameter of the potential can be determined by an appropriate configurational average at a previously determined melting point. The method is generated by the integration of a generalized Gibbs-Duhem equation and the steps are analogous to the derivation of the familiar Clapeyron equation for the slope of the $P-T$ coexistence curve. Gibbs-Duhem integration has been shown to be quite successful in efficiently determining the coexistence conditions for entire classes of potentials. For example, the phase diagram for the class of repulsive inverse power potentials, $u(r)=\epsilon(\sigma / r)^{n}$, was determined ${ }^{6}$ by starting with the known hard-sphere $(n=\infty)$ coexistence and integrating the derivative of coexistence curve with respect to the parameter $s \equiv 1 / n$. The method has also proved useful in a variety of other applications. ${ }^{7-9}$

In the current application, one begins with a model potential, parametrized for a real system in the usual way with respect to mechanical properties of the real system. The melting temperature (or pressure) for the model system is then calculated by thermodynamic integration. Once this is done, the derivative of the melting temperature (or pressure) with respect to all parameters of the system can be determined via separate simulations on the coexisting fluid and solid using the Gibbs-Duhem procedure. The calculated derivatives allow an assessment of the effect of each individual parameter on the melting point. From this information an appropriate scheme to adjust the parameters to improve the melting point can be devised in such a way that the agreement with the other experimental properties is not unacceptably compromised. The Gibbs-Duhem integration and our reparametrization scheme is outlined in more detail in the next section.

As a test application of this procedure, we examine an embedded atom model of aluminum developed by Mei and 
Davenport. ${ }^{3}$ This particular model was chosen for three reasons: First, the importance of aluminum as a material makes the development of an accurate model potential for simulation purposes desirable. Second, the large number of parameters and complicated nature of the embedded atom potential increases the need for a systematic, as opposed to ad hoc, procedure for adjusting the melting point. In addition, the zero-pressure melting point for the Mei-Davenport potential has been previously determined ${ }^{3}$ to be $800 \pm 9 \mathrm{~K}$, somewhat lower than the experimental melting point of aluminum at $933 \mathrm{~K}$. (Note that the melting point determined by Mei and Davenport was calculated for a 256-particle system - the actual value for this potential in the thermodynamic limit is slightly higher at $826.4 \pm 1.3 \mathrm{~K}$.) We find that, in this specific case, only one of the parameters of the potential has any significant effect on the potential and that changing this parameter according to the Gibbs-Duhem procedure yields a new model with the correct experimental melting point with no significant change in the quality of the agreement of the quantities with respect to which the model was originally optimized. Details of this calculation as well as a description of the model can be found in Sec. II below.

\section{GIBBS-DUHEM INTEGRATION AND MELTING POINT OPTIMIZATION}

The technique of Gibbs-Duhem integration has been well described previously by Kofke ${ }^{4}$, but in the interest of completeness and clarity we repeat the basic derivation here. Consider a single-component system with an arbitrary interaction potential $\mathcal{U}\left(\left\{\mathbf{R}_{i}\right\},\left\{X_{i}\right\}\right)$, where the $\mathbf{R}_{i}$ are the atomic coordinates and the $X_{i}$ are the parameters that define the potential—no restriction to pairwise additivity need be assumed. Assume there are two phases $\alpha$ and $\beta$ in coexistence at a temperature $T$ and pressure $P$. On the surface of coexistence, the chemical potentials (molar Gibbs free energies) of the two phases must be equal. To quantify how changes in $P, T$, and $X_{i}$ will affect the chemical potential one can define a generalized Gibbs-Duhem equation

$$
d \mu=-s d T+v d P+\sum_{i} \lambda_{i} d X_{i},
$$

where $\mu$ is the chemical potential, $s$ and $v$ are the entropy and volume per particle, respectively, and the $\lambda_{i}$ are generalized thermodynamic variables conjugate to the potential parameters $X_{i}$, defined as

$$
N \lambda_{i} \equiv\left(\frac{\partial G}{\partial X_{i}}\right)_{T, P, X_{j \neq i}} .
$$

Now as one moves infinitesimally away from the original coexistence point $\left(P, T,\left\{\lambda_{i}\right\}\right)$ to another point $(P+d P, T$ $\left.+d T,\left\{\lambda_{i}+d \lambda_{i}\right\}\right)$ on the surface of coexistence, the change in $\mu$ must be identical in both phases. This condition together with Eq. (1) gives

$$
\begin{aligned}
\mu_{\alpha}-\mu_{\beta}= & -\left(s_{\alpha}-s_{\beta}\right) d T+\left(v_{\alpha}-v_{\beta}\right) d P \\
& +\sum_{i}\left(\lambda_{\alpha, i}-\lambda_{\beta, i}\right) d X_{i}=0,
\end{aligned}
$$

where $\mu_{\alpha}$ and $\mu_{\beta}$ are the chemical potentials for each of the respective phases. Assuming constant pressure $(d P=0)$, since we are interested here in changes in the transition temperature, the previous equation can be rearranged to give

$$
\left(\frac{\partial T}{\partial X_{i}}\right)_{P, X_{j \neq i} ; \operatorname{coex}}=\frac{T\left(\lambda_{\alpha i}-\lambda_{\beta i}\right)}{h_{\alpha}-h_{\beta}}=\frac{T \Delta \lambda_{i}}{\Delta h},
$$

where we have also assumed that at coexistence, $\Delta s$ $=\Delta h / T$, where $\Delta h$ is the latent heat per particle for the phase transition. [Note that the corresponding equations for $\left(\partial P / \partial \lambda_{i}\right)_{T, \lambda_{j: j \neq i} ; \text { coex }}$ can be easily obtained by replacing $\Delta h / T$ in Eq. (5) with $\Delta v$.]

The $\lambda_{i}$ can be related to mechanical averages that can be easily calculated in a molecular dynamics or Monte Carlo simulation. First, the Gibbs free energy is related to the isothermal-isobaric distribution $\Delta(N, P, T)$ as follows:

$$
G=-k_{b} T \ln \Delta(N, P, T),
$$

which for a classical system with interaction potential $\mathcal{U}\left(\left\{\mathbf{r}_{i}\right\}\right)$ is given by

$$
\Delta(N, P, T)=\frac{1}{\Lambda^{3 N} N !} \int_{0}^{\infty} d V \int d^{N} \mathbf{r} \exp (-\beta \mathcal{U}-\beta P V),
$$

where $V$ is the volume. Taking the derivative of Eq. (6) with respect to the parameter $X_{i}$ gives

$$
\begin{aligned}
\left(\frac{\partial G}{\partial X_{i}}\right)_{T, P, X_{j \neq i}}= & -k T\left(\frac{\partial \ln \Delta}{\partial X_{i}}\right)_{T, P, X_{j \neq i}} \\
= & \frac{1}{\Delta} \int_{0}^{\infty} d V \int d^{N} \mathbf{r}\left(\frac{\partial \mathcal{U}}{\partial X_{i}}\right)_{X_{j} \neq i} \\
& \times \exp (-\beta \mathcal{U}-\beta P V) .
\end{aligned}
$$

Using Eq. (2) we have

$$
\lambda_{i}=\left(\frac{d G}{d X_{i}}\right)_{T, P, X_{j \neq i}}=\left\langle\left(\frac{\partial \mathcal{U}}{\partial X_{i}}\right)_{X_{j \neq i}}\right\rangle_{N, P, T} .
$$

Using Eq. (5) and Eq. (10) we can calculate the change necessary in each of the parameters $\left\{X_{i}\right\}$ to alter the melting temperature of our interaction potential by some arbitrary amount. As long as the changes are not too large, the calculation can be greatly simplified by the assumption that the deviation remains linear:

$$
T_{m}\left(\left\{X_{i}\right\}\right)=T_{m, 0}+\sum_{i}\left(\frac{\partial T}{\partial X_{i}}\right)_{X_{j \neq i}, \text { coex }}\left(X_{i}-X_{i, 0}\right),
$$

where $T_{m, 0}$ is the melting point of the original model with parameters $\left\{X_{i, 0}\right\}$. (If the linear approximation does not hold — and it should always be checked—one could integrate the differential equation using an appropriate numerical technique.) From a single simulation, one could calculate the necessary change in all the different parameters of the potential corresponding to a particular change in the melting temperature. One could then use this information to construct a 
TABLE I. Parameters for the aluminum embedded atom potential developed by Mei and Davenport. $E_{c}$ and $\phi_{0}$ are in units of eV. $r_{0}$ is in units of $\AA$, and the other parameters are dimensionless.

\begin{tabular}{lcccccc}
\hline \hline$E_{c}$ & $\phi_{0}$ & $r_{0}$ & $\alpha$ & $\beta$ & $\gamma$ & $\delta$ \\
3.39 & 0.1318 & 2.8638 & 4.60 & 7.10 & 7.34759 & 7.35 \\
$c_{0}$ & $c_{1}$ & $c_{2}$ & $c_{3}$ & $c_{4}$ & $c_{5}$ & \\
0.64085 & -6.83764 & 26.75616 & -47.16495 & 36.18925 & -8.60834 & \\
\hline
\end{tabular}

cost function to reoptimize the potential that includes the previously used set $\left\{A_{j}^{\text {expt }}\right\}$ of experimentally determined (mechanical) properties as well as the experimental melting temperature $T_{m}^{\text {expt }}$ as optimization targets. For example, a linear least squares procedure could be utilized using an appropriately chosen set of weight functions $w_{i}$ :

$f\left(\left\{X_{i}\right\}\right)=w_{T}\left[T_{m}^{\mathrm{expt}}-T_{m}\left(\left\{X_{i}\right\}\right)\right]^{2}+\sum_{j} w_{j}\left[A_{j}^{\mathrm{expt}}-A_{j}\left(\left\{X_{i}\right\}\right)\right]^{2}$.

(In the example application discussed in the next section, we found that only one of the several parameters of the potential had any significant effect on the melting point, which greatly simplified the reparametrization by making it possible to modify the potential without having to directly use such a cost function. It is not expected, however, that this will be true, in general.)

\section{RESULTS FOR A MODEL OF ALUMINUM: DETERMINING THE ORIGINAL MELTING POINT}

As an application of the method we examine an embedded atom model ${ }^{10}$ for aluminum developed by Mei and Davenport. ${ }^{3}$ The parameters in their potential were fit in order to optimize the potential with respect to a variety of experimental properties such as the cohesive energy $\left(E_{c}\right)$, lattice constant $\left(\sqrt{2} r_{0}\right)$, unrelaxed vacancy-formation energy, and elastic constants of the static fcc crystal at zero temperature. The total potential energy has the form

$$
\mathcal{U}=\sum_{i} F\left(\rho_{i}\right)+\frac{1}{2} \sum_{i, j \neq i} \phi\left(r_{i j}\right),
$$

where

$$
\begin{aligned}
F(\rho)= & -E_{c}\left[1-\frac{\alpha}{\beta} \ln \left(\frac{\rho}{\rho_{e}}\right)\right]\left(\frac{\rho}{\rho_{e}}\right)^{\alpha / \beta} \\
& +\frac{1}{2} \phi_{0} \sum_{m=1}^{3} s_{m} \exp [-(\sqrt{m}-1) \gamma] \\
& \times\left[1+(\sqrt{m}-1) \delta-\sqrt{m}\left(\frac{\delta}{\beta}\right) \ln \left(\frac{\rho}{\rho_{e}}\right)\right] \\
& \times\left(\frac{\rho}{\rho_{e}}\right)^{\sqrt{m} \gamma / \beta},
\end{aligned}
$$

and

$$
\begin{gathered}
\rho_{i}=\sum_{j(\neq i)} f\left(r_{i j}\right), \\
f(r)=\rho_{e} \sum_{l=0}^{5} \frac{c_{l}}{12}\left(\frac{r_{0}}{r}\right)^{l}, \\
\phi(r)=-\phi_{0}\left[1+\delta\left(\frac{r}{r_{0}}-1\right)\right] \exp \left[-\gamma\left(\frac{r}{r_{0}}-1\right)\right],
\end{gathered}
$$

and is used in conjunction with the following cutoff function:

$$
\begin{gathered}
q(r)= \begin{cases}1, & r \leqslant r_{n}, \\
(1-x)^{3}\left(1+3 x+6 x^{2}\right), & r_{n}<r<r_{c}, \\
0, & r \geqslant r_{c},\end{cases} \\
\quad x=\left(r-r_{n}\right) /\left(r_{c}-r_{n}\right),
\end{gathered}
$$

with a cutoff distance $\left(r_{c}\right)$ between the third- and fourthneighbor shells of a static fcc crystal. Using values of $r_{n}$ $=1.75 r_{0}$ and $r_{c}=1.95 r_{0}$, the functions $q(r) f(r)$ and

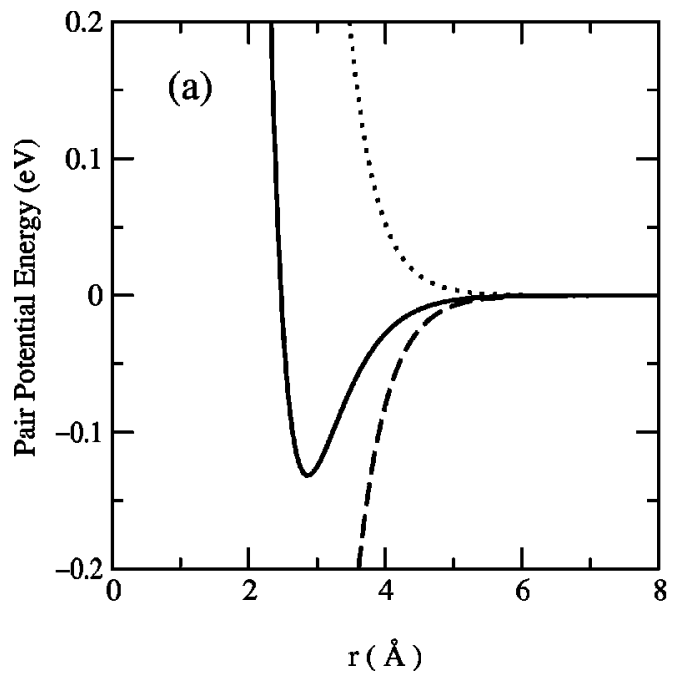

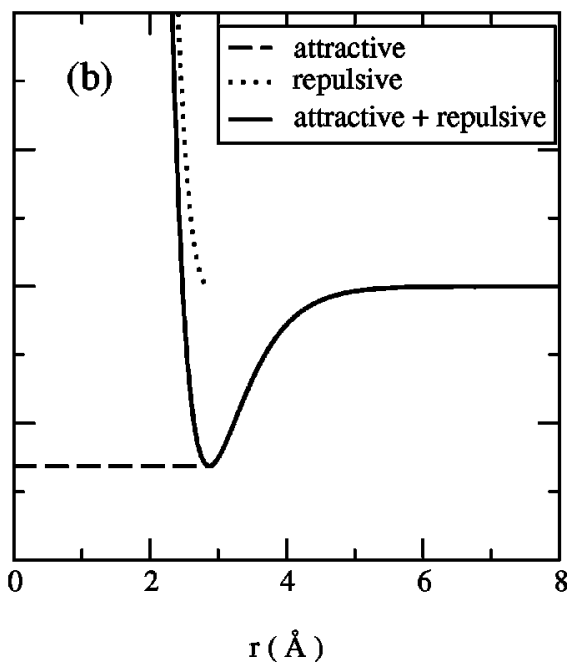

$r(\AA)$
FIG. 1. The different splitting methods for the pair part of the aluminum embedded atom potential are graphed separately as a function of $r$. (a) Mei-Davenport splitting and (b) WCA splitting methods. Note the difference in the effective radii between the combined and repulsive parts of the potential. In the MeiDavenport splitting this difference is large and can lead to freezing during the course of integration, whereas the WCA method has a much smaller difference in effective radii. 
TABLE II. Coefficients for the solid and liquid energy and density curves. The curves are polynomials of the form $y=a_{0} x^{2}+a_{1} x$ $+a_{2}$.

\begin{tabular}{lcccccc}
\hline \hline$N$ & & 256 & 500 & 864 & 2048 & 4000 \\
\hline Liquid energy & $a_{0}$ & $-3.95773 \times 10^{-8}$ & $-2.62765 \times 10^{-8}$ & $-3.47219 \times 10^{-8}$ & $-2.82724 \times 10^{-8}$ & $-3.14663 \times 10^{-8}$ \\
& $a_{1}$ & $4.24388 \times 10^{-4}$ & $3.98266 \times 10^{-4}$ & $4.15658 \times 10^{-4}$ & $4.02841 \times 10^{-4}$ & $4.08950 \times 10^{-4}$ \\
& $a_{2}$ & -3.39543 & -3.38194 & -3.39085 & -3.38448 & -3.38731 \\
Solid energy & $a_{0}$ & $5.75263 \times 10^{-8}$ & $5.85627 \times 10^{-8}$ & $5.88951 \times 10^{-8}$ & $5.88624 \times 10^{-8}$ & $5.88849 \times 10^{-8}$ \\
& $a_{1}$ & $2.30701 \times 10^{-4}$ & $2.30331 \times 10^{-4}$ & $2.30283 \times 10^{-4}$ & $2.30528 \times 10^{-4}$ & $2.30565 \times 10^{-4}$ \\
& $a_{2}$ & -3.38550 & -3.38533 & -3.38529 & -3.38532 & -3.38531 \\
Liquid density & $a_{0}$ & $7.59175 \times 10^{-10}$ & $1.61803 \times 10^{-10}$ & $5.35909 \times 10^{-10}$ & $3.19601 \times 10^{-10}$ & $3.91853 \times 10^{-10}$ \\
& $a_{1}$ & $-1.03177 \times 10^{-5}$ & $-9.15213 \times 10^{-6}$ & $-9.91989 \times 10^{-6}$ & $-9.47974 \times 10^{-6}$ & $-9.62320 \times 10^{-6}$ \\
& $a_{2}$ & $6.09309 \times 10^{-2}$ & $6.03495 \times 10^{-2}$ & $6.07444 \times 10^{-2}$ & $6.05232 \times 10^{-2}$ & $6.05920 \times 10^{-2}$ \\
Solid density & $a_{0}$ & $-2.59267 \times 10^{-9}$ & $-2.62088 \times 10^{-9}$ & $-2.63749 \times 10^{-9}$ & $-2.64149 \times 10^{-9}$ & $-2.64086 \times 10^{-9}$ \\
& $a_{1}$ & $-2.79768 \times 10^{-6}$ & $-2.78739 \times 10^{-6}$ & $-2.77752 \times 10^{-6}$ & $-2.77869 \times 10^{-6}$ & $-2.78125 \times 10^{-6}$ \\
& $a_{2}$ & $6.00427 \times 10^{-2}$ & $6.00404 \times 10^{-2}$ & $6.00379 \times 10^{-2}$ & $6.00380 \times 10^{-2}$ & $6.00387 \times 10^{-2}$ \\
\hline \hline
\end{tabular}

$q(r) \phi(r)$ go smoothly to zero at $r=r_{c}$. The parameters of the potential ${ }^{3,11}$ are given in Table I. For this embedded atom model, mass is measured in amu, distance in $\AA$, and energy in $\mathrm{eV}$. The natural simulation time unit is calculated to be 10.181 fs.

To begin, the melting temperature of the original model (at $P=0$ ) must be determined. Mei and Davenport performed a calculation of the free energies of the aluminum melt and fcc crystal using thermodynamic integration of the Gibbs free energy. For a 256-particle system, they obtain a melting temperature of $800 \pm 9 \mathrm{~K}$.

In repeating their melting point determination, we found that the value they obtained is not quite correct, due primarily to the small system size studied and a problem in the choice of thermodynamic integration path for the liquid phase. Our melting point determination was performed using the same basic methodology as Mei and Davenport, described below.

The Gibbs free energies per particle of the liquid and crystal as a function of temperature at constant pressure can be obtained by thermodynamic integration using the integral form of the Gibbs-Helmholtz equation

$$
\frac{g(T, P)}{T}=\frac{g\left(T_{0}, P\right)}{T_{0}}-\int_{T_{0}}^{T} \frac{h(\tau, P)}{\tau^{2}} d \tau,
$$

where $T_{0}$ is a predetermined reference temperature and $h(T, P)$ is the average total enthalpy per particle, which for $P=0$ is equal to the average total energy, $e(T, P=0)$. The Gibbs free energy at the reference temperature must be obtained separately by thermodynamic integration from a suitable ideal reference state. To do this, the interaction potential is parametrized along a linear path between that of the reference potential $\mathcal{U}_{0}$ and the full potential $\mathcal{U}$ :

$$
\mathcal{U}(\xi)=\xi \mathcal{U}+(1-\xi) \mathcal{U}_{0}
$$

The Gibbs free energy per particle relative to that of the reference system can then be obtained by thermodynamic integration along the path:

$$
\begin{aligned}
g(T, P= & 0) \equiv g(T, 0 ; \xi=1)=g\left(T_{0}, 0 ; \xi=0\right) \\
& +\int_{0}^{1} d \xi\left\langle\frac{\partial e(\xi)}{\partial \xi}\right\rangle_{\xi} .
\end{aligned}
$$

For the crystal, the reference system chosen is that of an Einstein crystal, ${ }^{12}$

$$
\mathcal{U}_{E}\left(\left\{r_{i}\right\}\right)=\frac{1}{2} m \omega_{D}^{2} \sum_{i}\left(\mathbf{r}_{i}-\mathbf{r}_{i 0}\right)^{2}
$$

where the $\left\{\mathbf{r}_{i 0}\right\}$ are the ideal crystal lattice positions, $m$ is the mass, and $\omega_{D}$ is the Debye frequency, which to minimize the difference between the reference and full system should be chosen to give a similar mean-squared displacement for the atoms at the temperature of interest. For this system at 296

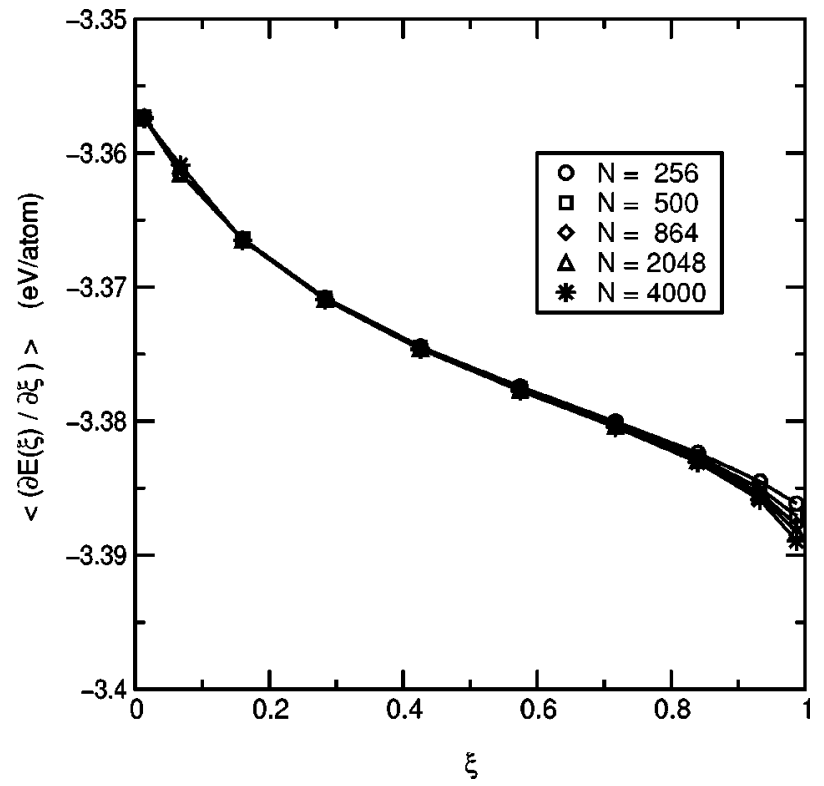

FIG. 2. Integrand for the numerical integration for the solid free energy at $296 \mathrm{~K}$ as a function of $\xi$. Values of $\xi$ were chosen based on the ten-point Gauss-Legendre quadrature. As the value of $\xi$ changes from 1 to 0 , the system is transformed from an embedded atom solid to that of an Einstein crystal. 

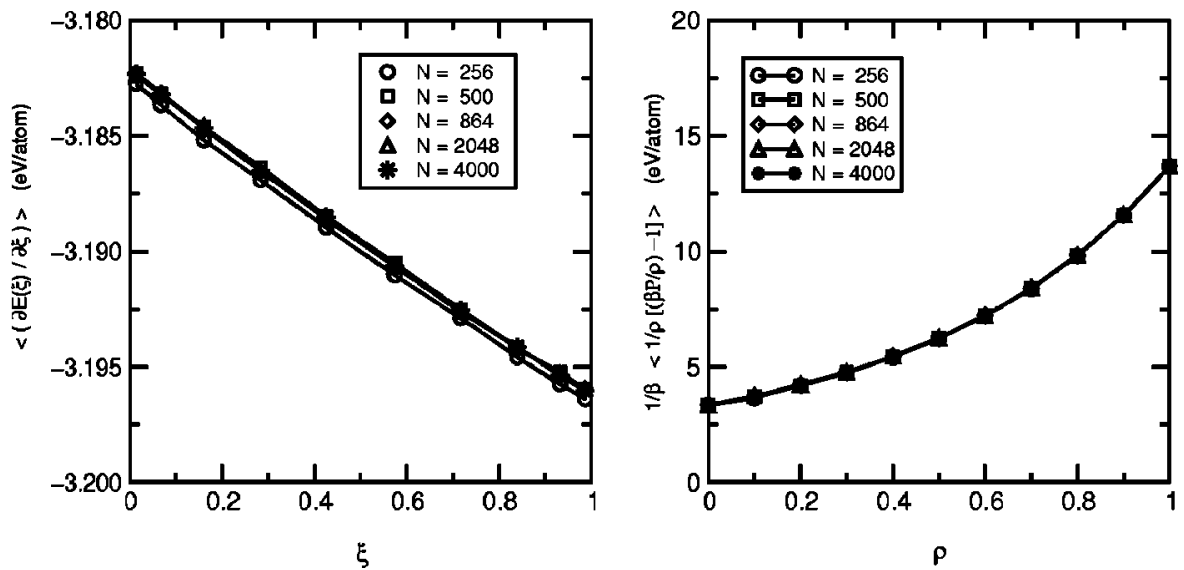

FIG. 3. (a) Simulation results for the integrand of step 1 of the liquid free energy calculation at $T=1092 \mathrm{~K}$ as a function of $\xi$. This integration slowly turns off the attractive part of the potential as the value of $\xi$ changes from 1 to 0 . Values for $\xi$ were again based upon the ten-point Gauss-Legendre quadrature. (b) Integrand for the volume expansion integration (step 2) in the liquid free energy calculation at $T=1092 \mathrm{~K}$ as a function of $\rho^{*}=\rho / \rho_{0}$. The numerical integration for this step was performed using the ten-point Simpson quadrature. The value at $\rho^{*}=0$ was obtained by an analytic calculation of the second virial coefficient $\left[B_{2}(T)\right]$.

$\mathrm{K}$, the optimum Debye frequency corresponds to a Debye temperature $\left(T_{D}=\hbar \omega_{D} / k\right)$ of $207 \mathrm{~K}$. Note that this frequency is different than the one used by Mei and Davenport.

The reference system for the liquid phase is an ideal gas, but the transformation must performed as a two-step process in order to avoid the liquid-gas phase transition. Mei and Davenport follow the reversible expansion method used by Broughton and Li. ${ }^{13}$ The two-step process is accomplished by first turning off the attractive part of the potential followed by a volume expansion to reach the ideal gas limit. In step 1 , it is extremely important to turn off the attractive part of the potential in a way that will not drastically alter the effective radius of the potential. If care is not taken, the system will freeze during the integration of this step. Here, we write the interatomic potential as a linear interpolation between the actual potential and the reference system:

$$
\phi(r ; \zeta)=\phi_{r e p}(r)+\zeta \phi_{a t t}(r)
$$

As the value of $\zeta$ is varied from 1 to 0 , the system is transformed from the original Mei-Davenport potential to that of a purely repulsive potential. Step 2 of the integration is a volume expansion. The free energy change in this step is given by

$$
\Delta g_{\text {step } 2}=k_{b} T_{0} \int_{0}^{\rho} \frac{d \rho^{\prime}}{\rho^{\prime}}\left[\frac{\beta P}{\rho^{\prime}}-1\right] .
$$

The potential splitting that Mei and Davenport use has a problem in that their repulsive potential $\phi_{\text {rep }}=\phi_{0} \delta \exp$ $\left[-\gamma\left(r / r_{0}-1\right)\right]$ has an effective radius that is much larger than the actual potential [see Fig. 1(a)] so that as the rest of the potential is turned off the system freezes as the effective packing fraction increases. To remedy this we use a Weeks-Chandler-Anderson ${ }^{14}$ (WCA) splitting where the repulsive part of the potential is equal to the potential energy for radii less than the radius at the minimum of the potential and zero for larger radii. This prescription (illustrated in Fig. 1) gives an effective radius similar to the full system and avoids the freezing transition.

In order to obtain energy curves needed in Eq. (18) as a function of temperature, molecular dynamics (MD) simulations were conducted at several different temperatures using the Nosé-Poincaré-Anderson algorithm ${ }^{15}$ for isothermalisobaric molecular dynamics. The fcc crystal was simulated at $50-\mathrm{K}$ intervals from 296 to $946 \mathrm{~K}$, while the liquid was studied over a smaller temperature range from 762 to $1152 \mathrm{~K}$ using 30-K intervals. All of the isothermal-isobaric MD simulation runs were equilibrated for 100000 steps and sampled for 300000 steps. From these simulations we ob-

TABLE III. Simulation averages for the solid and liquid Gibbs free energies along with the calculated melting temperature for several system sizes. Free energies are in units of eV/atom and the melting temperature is in $\mathrm{K}$.

\begin{tabular}{lccccc}
\hline \hline$N$ & 256 & 500 & 864 & 2048 & 4000 \\
Original $\delta$ & 7.35 & 7.35 & 7.35 & 7.35 & $-3.40254(1)$ \\
$g_{s}(T=296 \mathrm{~K})(\mathrm{eV} /$ atom $)$ & $-3.40200(3)$ & $-3.40219(2)$ & $-3.40236(2)$ & $-3.40246(1)$ & $-3.8565(2)$ \\
$g_{l}(T=1092 \mathrm{~K}(\mathrm{eV} /$ atom $)$ & $-3.8561(6)$ & $-3.8561(4)$ & $-3.8563(3)$ & $-3.8565(2)$ & $825.2 \pm 1.3$ \\
$T_{m}(\mathrm{~K})$ & $802.8 \pm 5.6$ & $814.3 \pm 3.7$ & $819.7 \pm 2.9$ & $822.5 \pm 1.9$ & 8.47 \\
New $\delta ;$ & 8.70 & 8.57 & 8.50 & $-3.39687(1)$ & $-3.39704(1)$ \\
\hline$g_{s}(T=296 \mathrm{~K}(\mathrm{eV} /$ atom $)$ & $-3.39538(3)$ & $-3.39615(2)$ & $-3.39664(1)$ & $-3.8209(2)$ & $-3.8217(2)$ \\
$g_{l}(T=1092 \mathrm{~K}(\mathrm{eV} /$ atom $)$ & $-3.8135(6)$ & $-3.8175(5)$ & $-3.8200(3)$ & $931.9 \pm 2.0$ & $931.5 \pm 1.5$ \\
$T_{m}(\mathrm{~K})$ & $934.9 \pm 5.9$ & $933.1 \pm 4.4$ & $930.9 \pm 3.0$ & & \\
\hline \hline
\end{tabular}


tained the average energy and density for both the crystal and liquid as a function of temperature and system size. Both the energies and densities were fit to second-order polynomials. The coefficients for these polynomials are shown in Table II. These polynomials were used in the construction of the free energy curves as described in Eq. (18).

Using the density obtained from the constant $N P T$ simulations, the Gibbs free energy of the fcc crystal at $T_{0}=296 \mathrm{~K}$ was calculated by running several simulations at constant $N V T$ using the Einstein crystal reference state to evaluate the integrand of Eq. (21) at values of $\xi$ corresponding to a tenpoint Gauss-Legendre quadrature. The simulations were performed using the Nosé-Poincaré algorithm. ${ }^{16}$ Figure 2 shows a plot of the integrand vs $\xi$ for all of the system sizes studied. At a value of $\xi=1$ the system is governed solely by the embedded atom potential. As the value of $\xi$ goes to zero, the potential is gradually changed to that of an Einstein crystal. For each value of $\xi$, the system was equilibrated for 100000 steps and sampled for 100000 steps.

Simulations for step 1 of the liquid free energy at a temperature of $1092 \mathrm{~K}$ were performed in the same manner as for the crystal. Here the attractive part of the potential [Eq. (22)] was slowly turned off as the value $\xi$ was changed from 1 to 0 . This is shown graphically in Fig. 3(a). As with the solid, ten-point Gauss-Legendre integration was used to numerically compute this integral. The second step of the liquid free-energy calculation included a series of constant $N V T$ simulations at decreasing densities starting with the repulsive potential system from the conclusion of step 1. Average values for the step-2 integrand [Eq. (24)] are shown graphically in Fig. 3(b). The simulations for both steps of the liquid free-energy integrations were equilibrated for 100000 steps and sampled for 100000 steps at each value of the integrand. The numerical integration for step 2 was performed using the ten-point Simpsons quadrature. This method was chosen over the Gauss-Legendre quadrature due to the inaccuracy of the sampling at low density. The value of the integrand at zero density was obtained by an analytical calculation of the second virial coefficient $\left(B_{2}\right)$.

Free energies and melting points are shown in Table III as a function of particle number. A graph of the melting temperature vs $1 / N$ (Fig. 4) shows that at infinite particle number the melting point for the embedded atom potential proposed by Mei and Davenport approaches $826.4 \pm 1.3 \mathrm{~K}$. It should be noted that the major error in the melting point calculated by Mei and Davenport for their potential $800 \pm 9 \mathrm{~K}$ is primarily due to the small size of their system. The problems with their potential splitting appears to have had little effect, probably due to cancellation of errors, as the melting point that we determine here for the 256-particle system agrees with theirs within the simulation error. Recently, Morris et al. ${ }^{17}$ argued on the basis of the stability of crystal-liquid interfaces that the melting point of the Mei-Davenport aluminum potential is actually significantly lower (around $725 \mathrm{~K}$ ). Our results do not support this conclusion and as a check we have carefully set up stable stress-free interfaces at our calculated melting point. The systems exhibit melting (freezing) as the temperature is raised (lowered) away from our calculated melting point. The lower-temperature transitions of Morris et al. were most likely due to significant unrelaxed stress in the crystal.

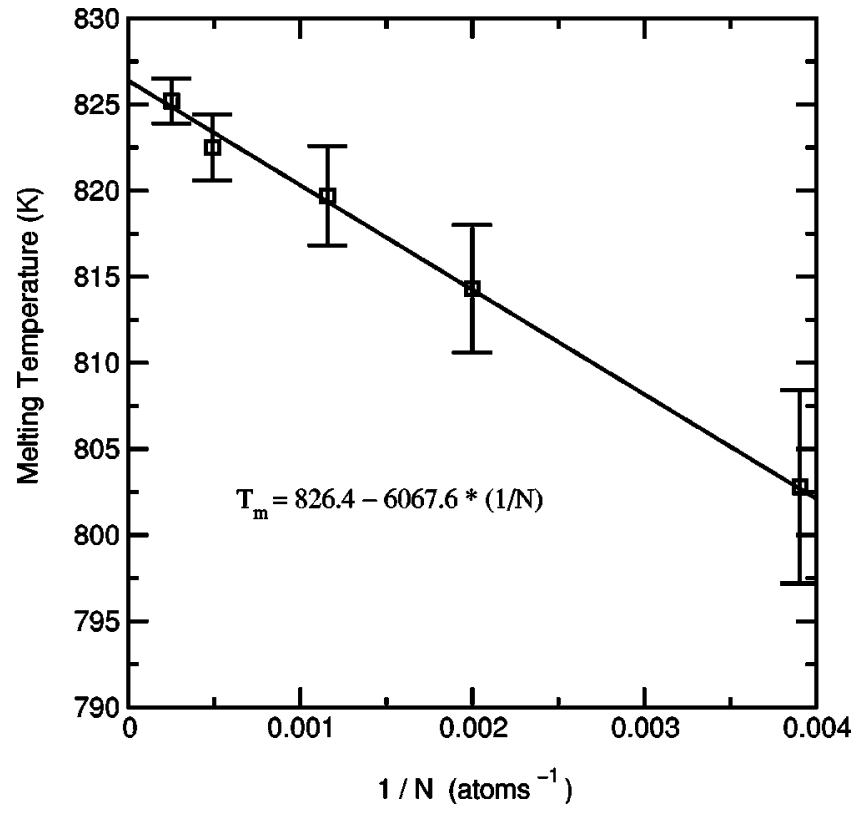

FIG. 4. The melting temperature of the embedded atom potential is plotted as a function of inverse particle size. As $N$ goes to infinity $(1 / N \rightarrow 0)$, the melting temperature approaches $826.4 \mathrm{~K}$.

\section{RESULTS FOR A MODEL OF ALUMINUM: REPARAMETRIZING THE POTENTIAL}

To adjust the melting temperature of the embedded atom potential for aluminum, we first calculate $\partial T_{m} / \partial X_{i}$ for each of the parameters in the potential. [Note that, for simplicity and consistency, the expansion coefficients $c_{l}$ in Eq. (14) were kept constant.] The derivatives were calculated in a single (constant $N P T$ ) simulation at the melting temperature calculated in the previous section with $P=0$, for each of several system sizes $(N=256,500,864,2048$, and 4000).

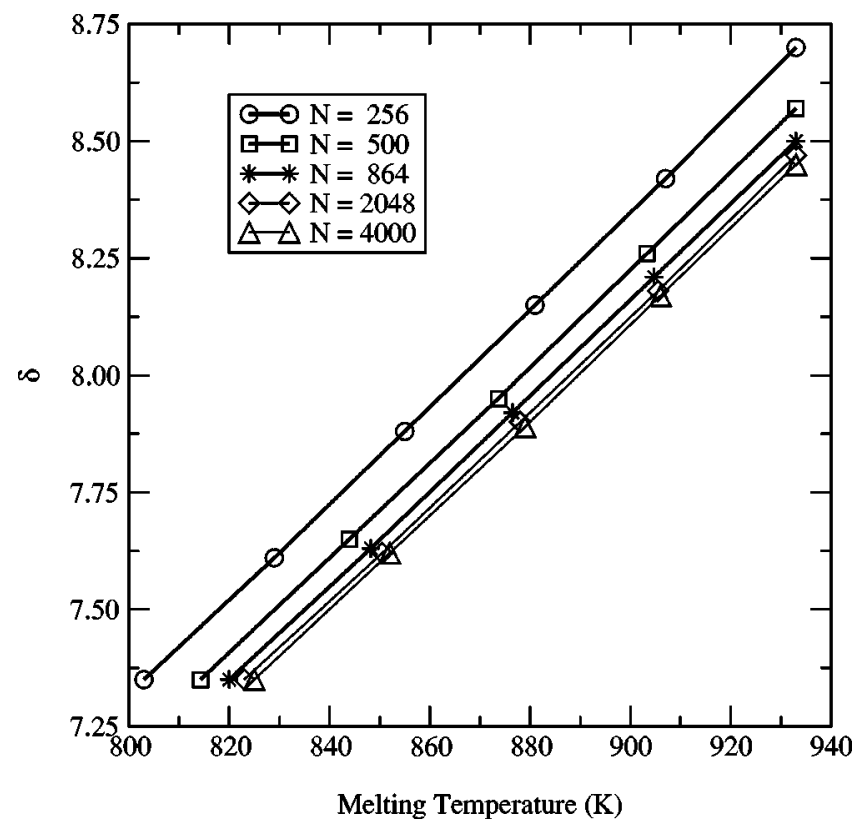

FIG. 5. Values of $\delta$ along the Gibbs-Duhem integration of the crystal-melt coexistence curve. These curves were generated using a fourth-order predictor corrector and show the melting temperature as a function of $\delta$ for each of the various system sizes. 
TABLE IV. Here the vacancy formation energy, latent heat values, and elastic constants are presented for the original version of the EAM Al potential and the modified version. $C_{11}$ and $C_{12}$ for the modified version (with the experimental melting temperature) are closer to their experimental values. $C_{44}$ seems to get slightly worse.

\begin{tabular}{lccc}
\hline \hline & $\delta=7.35$ & $\delta=8.45$ & Experimental \\
\hline Unrelaxed vacancy formation (eV/atom) & 4.07211 & 4.18679 & N/A \\
Latent heat (eV/atom) & 0.0830 & 0.0973 & 0.111 \\
$C_{11}\left(\mathrm{~J} / \mathrm{m}^{3}\right)$ & 0.093 & 0.096 & 0.107 \\
$C_{12}\left(\mathrm{~J} / \mathrm{m}^{3}\right)$ & 0.069 & 0.068 & 0.061 \\
$C_{44}\left(\mathrm{~J} / \mathrm{m}^{3}\right)$ & 0.033 & 0.036 & 0.028 \\
\hline \hline
\end{tabular}

The system was equilibrated for 100000 steps followed by 100000 steps for averaging. From this simulation it was determined that $\partial T_{m} / \partial X_{i}$ was significant only for the parameter $\delta$ - the other parameters of the potential have little effect on the melting point. The complicated nature of the potential makes it difficult to assign any physical explanation to the sensitivity of the melting point to $\delta$ relative to the other parameters.

Next, a series of simulations were performed for each system size to integrate along the coexistence curve from the initial calculated melting point of the potential to the true experimental melting temperature. At each temperature along the coexistence curve, the system was equilibrated for 100000 steps and sampled over 300000 steps. In our experiments we use a fourth-order predictor corrector integrator to carry out the integration along the coexistence curve as a function of $\delta$. Figure 5 shows this integration graphically with final values of $\delta$ corresponding to a melting temperature of $T_{m}=933 \mathrm{~K}$. These values are listed in Table III. (Note that, although we did an accurate numerical integration along the coexistence curve, the results indicate that using the approximation that the derivative is a constant in the region of interest would have been correct to within the simulation error.)

In order to confirm that the melting point did indeed change as expected, the melting point calculation was repeated using the newly calculated value of $\delta$. The new melting temperature for the embedded atom potential with a value of $\delta=8.45$ corresponding to a system size of $\mathrm{N}=4000$ was calculated to be $931.5 \pm 1.5 \mathrm{~K}$. Melting temperatures for the other system sizes are listed in Table III. The experimental value for the melting temperature of aluminum is 933.47 $\mathrm{K}$.

Mei and Davenport initially determined $\delta$ (and the other parameters in the potential) by fitting the potential to certain physical constants. For the new value of $\delta$ (8.45), we have recalculated a variety of physical properties of aluminum. These quantities, for the original potential, the reparametrized potential, and the experimental values, are collected in Table IV. From these data, we see that, in comparison to the original potential with $\delta=7.35$, the new potential more closely models the experimental values ${ }^{18}$ of the $(T=0 \mathrm{~K})$ elastic constants $C_{11}$ and $C_{22}$ while $C_{44}$ becomes slightly worse in comparison to its experimentally determined value. In addition, mostly as a consequence of the improved melting point, the latent heat is considerably improved.

\section{SUMMARY}

We have outlined an application of the Gibbs-Duhem integration method of Kofke, ${ }^{4}$ with which a model interaction potential can be reparametrized, including the experimental melting point in the optimization protocol. The melting temperature of a potential can then be adjusted similar to the tuning of other parameters in the potential. Since nonmechanical properties, such as the melting point, are not generally included in potential optimization and the agreement of such quantities with experiment is not guaranteed, such a procedure will be useful in situations, such as in the simulation of crystal-melt interfaces, where having the correct melting point is highly desirable. The method is general and can easily be extended to a variety of systems. As an example of the utility of the method, we apply the procedure to reparametrizing a popular model of aluminum ${ }^{3}$ for which the melting point has been calculated to be over $100 \mathrm{~K}$ below the accepted experimental value. We demonstrate that the reparamtrized potential has a melting point that agrees within the statistical error with the experimental value of $933 \mathrm{~K}$ and that reparametrization does not degrade the quality of the potential with respect to a variety of properties, and that, in fact, for quantities such as the elastic constants $C_{11}$ and $C_{12}$ and the latent heat, agreement is improved.

\section{ACKNOWLEDGMENTS}

We gratefully acknowledge J. W. Davenport for helpful conversations, as well as the Kansas Center for Advanced Scientific Computing for the use of their computer facilities. We also would like to thank the National Science Foundation (under Grant No. CHE-9500211) as well as the University of Kansas General Research Fund for their generous support of this research.

\footnotetext{
*Author to whom correspondence should be addressed.

${ }^{1}$ B. B. Laird, in The Encyclopedia of Computational Chemistry, edited by P. v. R. Schleyer, N. L. Allinger, P. Kollman T. Clark, and H. F. Schaefer (Wiley, New York, 1998).
}

${ }^{2}$ J. R. Errington and A. Z. Panagiotopoulos, J. Chem. Phys. 111, 9731 (1999).

${ }^{3}$ J. Mei and J. W. Davenport, Phys. Rev. B 46, 21 (1992).

${ }^{4}$ D. A. Kofke, Mol. Phys. 78, 1331 (1993). 
${ }^{5}$ D.A. Kofke, J. Chem. Phys. 98, 4149 (1993).

${ }^{6}$ R. Agrawal and D. A. Kofke, Phys. Rev. Lett. 74, 122 (1995).

${ }^{7}$ M. H. J. Hagen, E. J. Meijer, G. C. A. M. Mooij, D. Frenkel, and H. N. W. Lekkerkerker, Nature (London) 365, 425 (1993).

${ }^{8}$ E. J. Meijer and D. Frenkel, J. Chem. Phys. 100, 6873 (1994).

${ }^{9}$ W. O. Haag, in Zeolites and Related Microporous Materials: State of the Art 1994, edited by J. Weitkamp (Elsevier Science, Oxford, 1994), Vol. 84, p. 1375.

${ }^{10}$ R. A. Johnson, Phys. Rev. B 37, 3924 (1988).

${ }^{11}$ J. Mei, J. W. Davenport, and G. W. Fernando, Phys. Rev. B 43, 4653 (1991).

${ }^{12}$ D. Frenkel and B. Smit, Understanding Molecular Simulation
(Academic Press, New York, 1996).

${ }^{13}$ J. Q. Broughton and X. P. Li, Phys. Rev. B 35, 9120 (1987).

${ }^{14}$ J. D. Weeks, D. Chandler, and H. C. Anderson, J. Chem. Phys. 54, 5237 (1971).

${ }^{15}$ J. B. Sturgeon and B. B. Laird, J. Chem. Phys. 112, 3474 (2000).

${ }^{16}$ Stephen D. Bond, Benedict J. Leimkuhler, and Brian B. Laird, J. Comput. Phys. 151, 114 (1999).

${ }^{17}$ J. R. Morris, C. Z. Wang, K. M. Ho, and C. T. Chan, Phys. Rev. B 49, 3109 (1994).

${ }^{18}$ N. W. Ashcroft and N. D. Mermin, Solid State Physics (CBS, Hong Kong, 1976). 